Nat. Hazards Earth Syst. Sci., 12, 3241-3253, 201
www.nat-hazards-earth-syst-sci.net/12/3241/2012
doi:10.5194/nhess-12-3241-2012
(C) Author(s) 2012. CC Attribution 3.0 License.

\author{
(1)
}

\title{
Definition of a shortcut methodology for assessing flood-related Na-Tech risk
}

\author{
E. Marzo, V. Busini, and R. Rota \\ Politecnico di Milano, Dept. Chemistry, Materials and Chemical Engineering “Giulio Natta”, Piazza Leonardo da Vinci 32, \\ 20133 Milano, Italy
}

Correspondence to: V. Busini (valentina.busini@polimi.it)

Received: 4 May 2012 - Revised: 17 September 2012 - Accepted: 18 September 2012 - Published: 6 November 2012

\begin{abstract}
In this paper a qualitative methodology for the initial assessment of flood-related Na-Tech risk was developed as a screening tool to identify which situations require a much more expensive quantitative risk analysis (QRA). Through the definition of some suitable key hazard indicators (KHIs), the proposed methodology allows the identification of the Na-Tech risk level associated with a given situation; the analytical hierarchy process (AHP) was used as a multicriteria decision tool for the evaluation of such qualitative KHIs. The developed methodology was validated through two case studies by comparing the predicted risk levels with the results of much more detailed QRAs previously presented in literature and then applied to the real flood happened at Spolana a.s., Neratovice, Czech Republic in August 2002.
\end{abstract}

\section{Introduction}

Natural disasters may be powerful and prominent mechanisms of direct or indirect release of hazardous material (Young et al., 2004). If industrial sites are located in naturally hazard-prone areas, technological accidents may be triggered by natural events, which could generate the so-called NaTech (Natural and Technological) events and may modify as well as increase the impact and overall damage in surrounding areas (Galderisi et al., 2008).

In recent years $\mathrm{Na}$-Tech events have received a significant attention and many reviews on Na-Tech events spanning over $30 \mathrm{yr}$ have been published (Chang and Lin, 2006; Cruz et al., 2006; Rasmussen, 1995; Showalter and Myers, 1994; Krausmann et al., 2011); nevertheless, there is little information available on the interactions between natural disasters and simultaneous technological accidents. In addition, natural disasters have increased both in frequency and economic losses around the world (Young et al., 2004); therefore, there is increasing public awareness and interest from the scientific community (Renni et al., 2010). Recent examples of Na-Tech events are reported in the literature (Cozzani et al., 2010; Cruz and Krausmann, 2009; Cruz and Okada, 2008; Picou, 2009), but only a few papers discuss approaches and methodologies necessary to face the problems they cause (Cozzani et al., 2006; Cruz and Okada, 2008; Fendler, 2008; Galderisi et al., 2008; Krausmann and Mushtaq, 2008).

The most powerful tool to evaluate the impact that a natural event may have on industrial facilities is an extension of the classical quantitative risk analysis (QRA) to situations where an industrial accident is triggered by a natural event (Antonioni et al., 2007, 2009; Campedel et al., 2008b; Fabbrocino et al., 2005). A limitation of the QRA is that it requires large amounts of resources in terms of both time and expertise; thus, a shortcut methodology for the assessment of industrial risks induced by natural events, which should be easy to handle and capable of taking into account the most important phenomena that occur in a Na-Tech event, should be useful at least for screening purposes (i.e. for deciding when it is worthwhile to conduct a QRA). In this work, a simple methodology for assessing qualitatively flood-related $\mathrm{Na}$-Tech risk was developed and validated by comparison with available results from more detailed QRAs.

\section{Methodology}

A shortcut procedure should be easy to apply, require a small amount of resources and information and summarize, through suitable qualitative key hazard indicators (KHIs), the 
Na-Tech risk level associated with a given situation (i.e. a process plant located at a given position).

Unfortunately, estimating the values of such KHIs requires a simultaneous comparisons among a large number of different parameters, ranging from the types of hazardous substances present in the plant to the intensity of the external natural force, which are incommensurable, i.e. not directly comparable due to different units of measure and physical meaning (Bell and Glade, 2004).

This requires the use of a multi-criteria decision method to account for the different and often incommensurable effects (heat radiation due to a fire, pressure waves due to an explosion, toxicity due to a dispersion of a toxic compound and so on) of various parameters. Among the various approaches available, the analytical hierarchy process (AHP) (Saaty, 2000) is a method that can support decision making by establishing alternatives within a framework of multiweighted criteria. This method allows for choosing among various alternatives through binary comparison, that is, considering only two elements at a time. The idea of using the AHP in the context of Na-Tech risk analysis has been recently proposed (Rota et al., 2008), and a practical shortcut procedure for earthquake-related $\mathrm{Na}-\mathrm{Tech}$ events has been developed (Busini et al., 2011). As for the earthquake-related $\mathrm{Na}-\mathrm{Tech}$ events, the use of the AHP requires the identification of all the main elements that can influence the vulnerability of the plant with respect to floods; such elements, while covering all the relevant aspects, should be few and easy to evaluate.

All the details of using the AHP for developing a shortcut methodology for assessing Na-Tech risk are not reported in the present paper, since they are extensively discussed elsewhere (Busini et al., 2011); here it suffices to mention that binary comparisons between elements must be established, and they must be arranged in suitable hierarchies structured with the goal on top (in this case the KHI), with different branches. At the bottom of the hierarchy there are the alternatives, which characterize the given plant with respect to the Na-Tech effect on people. In Na-Tech accidents, the main consequences are broadly due to three phenomena: fires, explosions, and toxic dispersions. Therefore, three different hierarchies must be developed for $\mathrm{Na}$-Tech accidents leading to three distinct $\mathrm{KHI}$ : $\mathrm{KHI}_{\mathrm{F}}, \mathrm{KHI}_{\mathrm{T}}$, and $\mathrm{KHI}_{\mathrm{E}}$, for fires, toxic dispersion, and explosion, respectively.

Through simple mathematical manipulations (Saaty, 2000), from the normalized values assigned to the alternatives, it is possible to compute the three KHIs values on a $0-1$ scale. To determine the overall risk level of a given plant characterized by the three values of $\mathrm{KHI}_{\mathrm{F}}, \mathrm{KHI}_{\mathrm{T}}$, and $\mathrm{KHI}_{\mathrm{E}}$, these values can be condensed into a global $\mathrm{KHI}\left(\mathrm{KHI}_{\mathrm{G}}\right)$, which represents the overall risk level in the KHIs space. Because the origin of the 3-D KHIs space represents the optimal condition, i.e. the lower the KHI value the lower the related risk level, a point into the KHIs space, identified by the three values $\mathrm{KHI}_{\mathrm{F}}, \mathrm{KHI}_{\mathrm{T}}$, and $\mathrm{KHI}_{\mathrm{E}}$, represents a risk level related to its distance from the origin. Therefore, $\mathrm{KHI}_{\mathrm{G}}$ can be evaluated through a norm providing the distance from the origin, which in a 3-D space can be simply obtained as the square root of the sum of the three squared KHIs (Busini et al., 2011):

The value of the $\mathrm{KHI}_{\mathrm{G}}$ can be related to different risk levels, reasonably indicating a low sensitivity to the Na-Tech events of the analyzed plant for $\mathrm{KHI}_{\mathrm{G}}<10^{-2}$, medium for $10^{-2}<\mathrm{KHI}_{\mathrm{G}}<10^{-1}$ and high for $\mathrm{KHI}_{\mathrm{G}}>10^{-1}$.

High rated plants require further analysis through a dedicated QRA, while low rated plants do not. The Na-Tech risk related to medium rated plants is neither negligible nor unacceptable; this is a sort of ALARP (As Low As Reasonably Practicable) region (Lees, 1996), where the decision is determined by the analyst and must be decided on a case-by-case basis.

\section{Hierarchies}

\subsection{Hierarchy for fires $\left(\mathrm{KHI}_{\mathrm{F}}\right)$}

The $\mathrm{KHI}_{\mathrm{F}}$ is related to the impact area of fires (e.g. fireball, flash fire, or pool fire) arising from a Na-Tech event.

Along the same lines discussed elsewhere (Busini et al., 2011), it can be assumed that the elements that primarily influence the impact area are the specific thermal power of the stored material and the specific flow of combustible vapors arising from the released materials. This leads to the hierarchy summarized in Fig. 1, which is composed by the fundamental objective $\left(\mathrm{KHI}_{\mathrm{F}}\right)$, two levels related to the two main elements identified (in terms of combustion enthalpy and volatility), and the alternatives representing the plant/territory characteristics. A given plant located in a specific environment subject to flooding risk is characterized by the ensemble of the basic alternatives. Each "alternative" is characterized by an overall normalized index, $\bar{M}_{h b}$ (where $h$ indicates the hierarchy $\mathrm{F}, \mathrm{T}$ or $\mathrm{E}$, while $b$ indicates a branch of a hierarchy) of the amount of a given class of substances which is expected to be released in case of a flood event with a given intensity. Clearly, a branch may not be utilized (the branch has zero weight in calculating the index), depending on the characteristics of the materials present in the considered plant. Analogously, one or two of the three hierarchies may be not used (e.g. in the case of toxic substances that are neither flammable nor explosive). On the other hand, a volatile flammable material characterized by toxic vapors will be the input of both the hierarchy for fires and the hierarchy for toxic dispersion.

The relative importance among the different branches of the same hierarchy were defined on the basis of technical rules of thumb as discussed elsewhere (Busini et al., 2011). Since the definition of the alternatives, which characterize a given plant in a specific environment, is common to the three hierarchies it will be discussed later. 


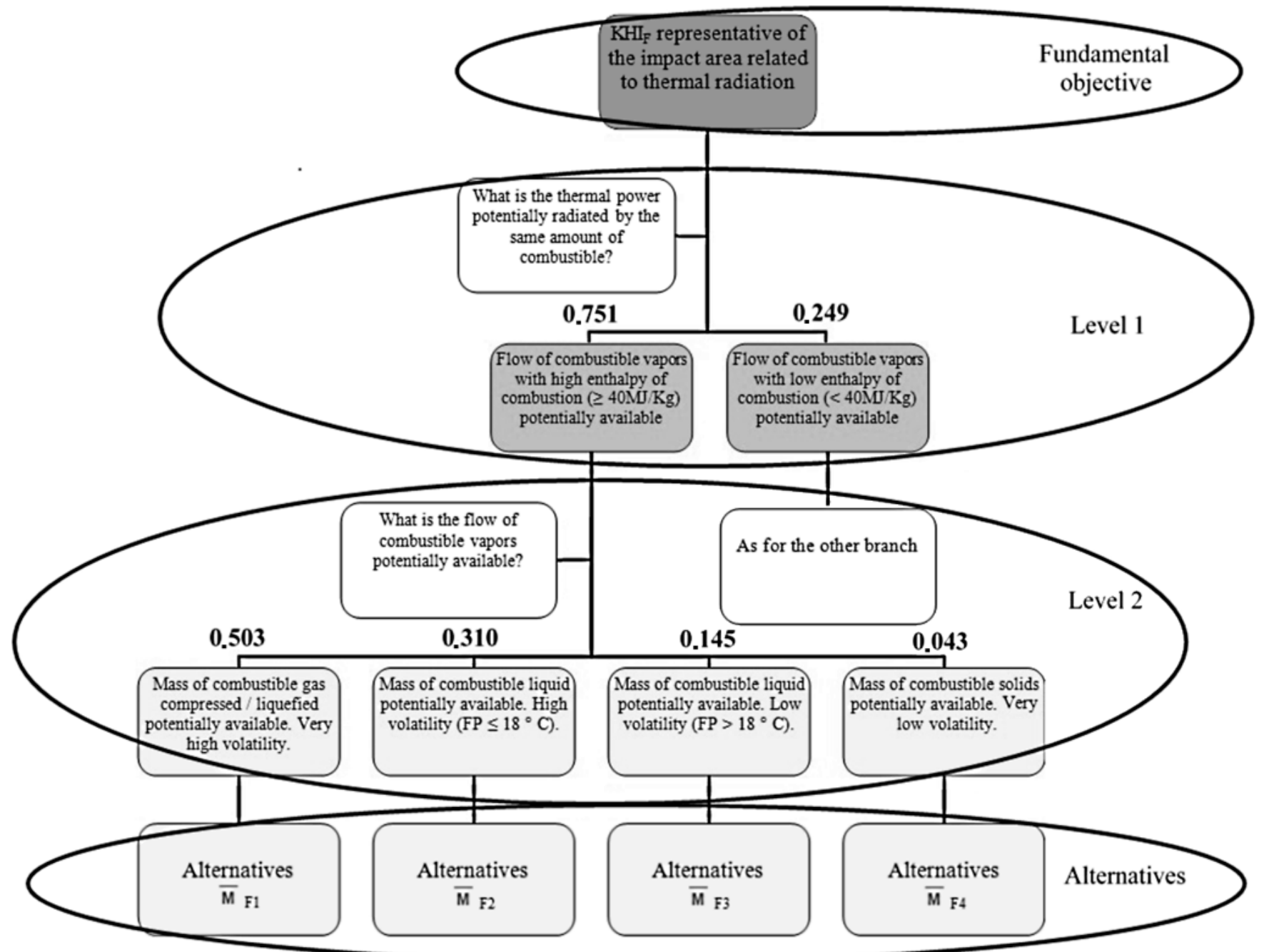

Fig. 1. Hierarchy used for flood-related $\mathrm{Na}$-Tech qualitative risk analysis in case of fires. The weights assigned are shown along the different branches, while the related question to be answered is located above each level.

\subsection{Hierarchy for toxic dispersion $\left(\mathrm{KHI}_{\mathrm{T}}\right)$}

$\mathrm{KHI}_{\mathrm{T}}$ is related to the impact area of a Na-Tech event related to the release of toxic materials that can disperse into the atmosphere.

In this case, the principal elements are the toxicity (summarized in the IDLH value) and the volatility of the released material. This leads to the hierarchy summarized in Fig. 2, which is constituted by the fundamental objective $\left(\mathrm{KHI}_{\mathrm{T}}\right)$, two levels related to the two main elements identified (toxicity and volatility), and the alternatives.

Also in this case, full details concerning the criteria and the technical considerations about the choice of the weights are reported elsewhere (Busini et al., 2011).

\subsection{Hierarchy of explosions $\left(\mathrm{KHI}_{\mathrm{E}}\right)$}

$\mathrm{KHI}_{\mathrm{E}}$ is related to the impact area of a Na-Tech event leading to an explosion; the principal elements correspond to the type of energy released (mechanical or chemical), the specific energy (in terms of pressure or combustion enthalpies), and the volatility (for vapor cloud explosion, VCE), as summarized in Fig. 3.

Criteria and technical considerations about the choice of the weights are discussed elsewhere (Busini et al., 2011).

\section{Evaluation of the alternatives: characteristics of the plant/flood}

For each branch a normalized index of the mass of similar materials (i.e. capable of generating similar scenarios in terms of effects), which can be released following a flood 


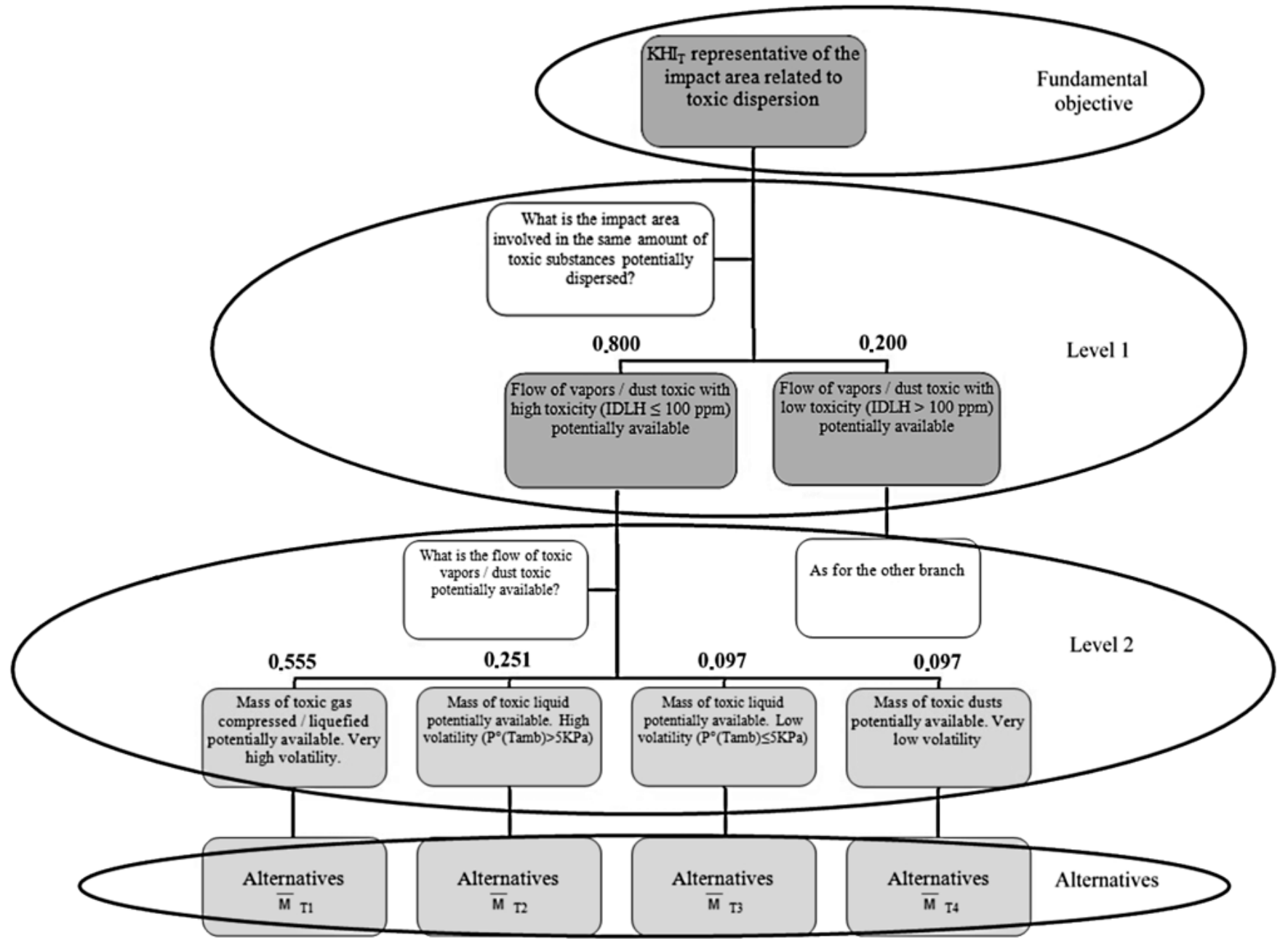

Fig. 2. Hierarchy used for the flood-related $\mathrm{Na}$-Tech qualitative risk analysis in case of a toxic dispersion. The weights assigned are shown along the different branches, while the related question to be answered is located above each level.

event having a certain intensity, should be provided. The procedure for the calculation of the normalized indexes $\bar{M}_{h b}(h$ corresponds to one of the three hierarchies T, $\mathrm{F}$, or $\mathrm{E}$ while $b$ corresponds to one of the branches of the hierarchy) is summarized in the block diagram of Fig. 4.

In literature there are no simple and general methods or parameters to evaluate and quantify the severity of floods, which are usually scheduled through their return period, usually between 10 and $500 \mathrm{yr}$. The return period $\left(T_{\mathrm{R}}\right)$ denotes the interval between two events of the same intensity and then indirectly expresses the probability that a flood will occur. Concerning the land use planning, usually fluvial regions (the so-called "fluvial strips"), which delimit the riverbed and identify floodplains, are defined (Autorità di bacino del fiume Po, 1999). The delimitation of areas of potential flooding in the event of failure of the banks is done through analysis of historical routes, morphological characteristics of the surrounding area and the hydraulic quantities involved (Autorità di bacino del fiume Po, 1999).
For instance, in Italian maps, areas of flooding as a function of the return time are identified through return period values (Presidenza del Consiglio dei Ministri, 29 settembre 1998 (G.U. 5 gennaio 1999 n.3)).

In particular three "fluvial strips" are identified: a "fluvial strip A" corresponding to the active river basin, an intermediate zone that includes the first and extends up to the banks ("fluvial strip B" with return period of $200 \mathrm{yr}$ ), and a third strip of extraordinary flooding that also includes the first two ("fluvial strip C" with a return period of $500 \mathrm{yr}$ ). The term active river basin is used to define that part of the territory affected by the flow of water or by the ordinary flood, and includes areas that belong morphologically to the river, separated from the edge of the bank or from the inner natural levee.

Criteria different from the aforementioned one can be used for defining the "fluvial strips" with respect to different values of the return period; however, we assume that at least a three level classification ("strip A", high probability of 


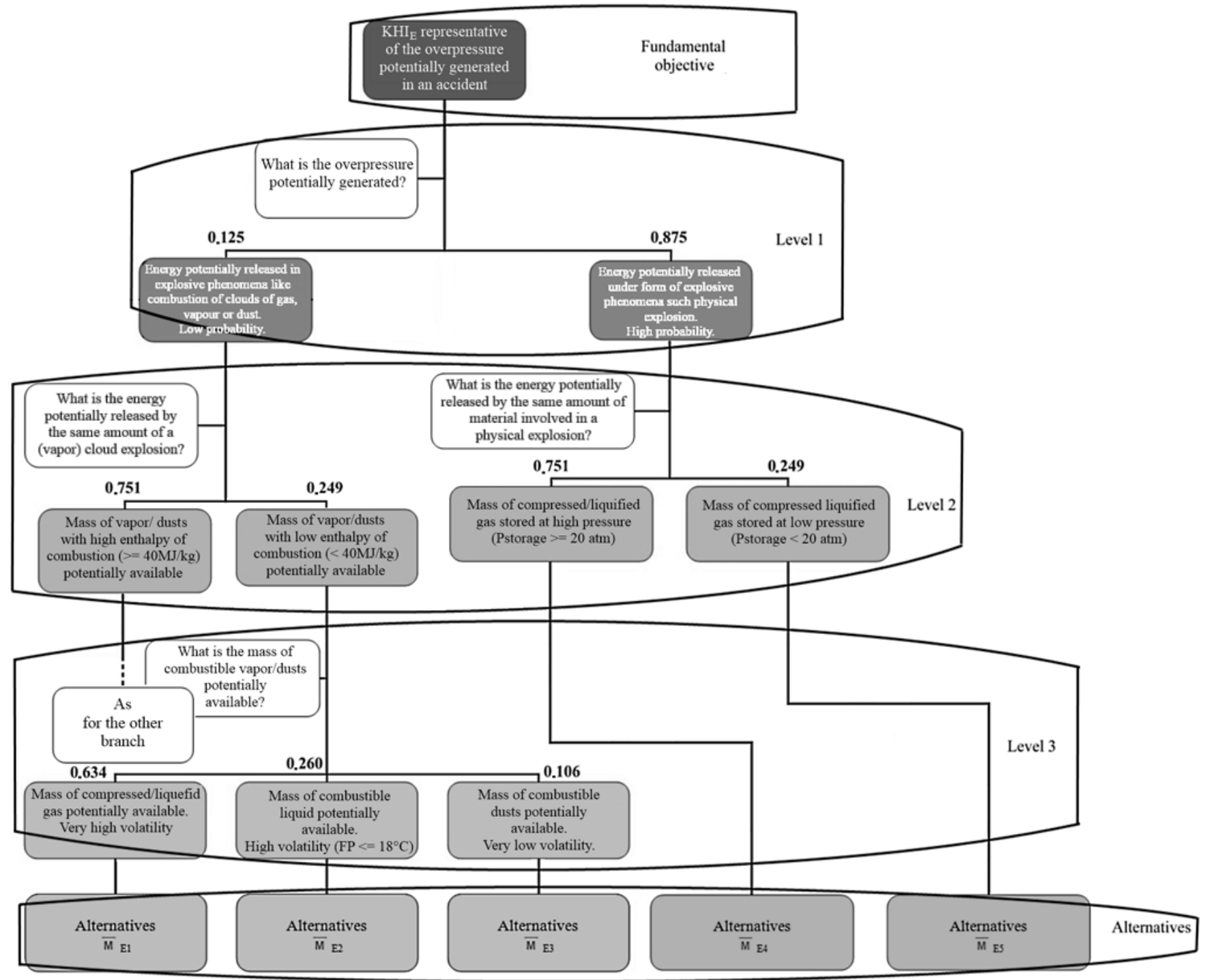

Fig. 3. Hierarchy used for flood-related Na-Tech qualitative risk analysis in case of an explosion. The weights assigned are shown along the different branches, while the related question to be answered is located above each level.

flooding; "strip B", medium probability and "strip C", low probability) is available with the corresponding return periods.

The first step in the procedure summarized in Fig. 4 is to identify the "fluvial strip" where the plant is located. Once the reference "fluvial strip" is known, the magnitude of the expected flood event in terms of height and velocity of the water has to be estimated; since data concerning expected water depth and water velocity, which of course would be the optimum, are not always available, a simplified procedure to estimate them was developed. It assumes that

- The maximum water depth $(\Delta Z)$ may be estimated as the difference between the quote of the boundary of the "fluvial strip" $\left(Z_{\mathrm{PAI}}\right)$, where the plant is located, and the lowest elevation in the site of interest $\left(Z_{\mathrm{i}}\right)$, that is, the lowest point of the plant. When the plant is located on a higher quote than the limit of the fluvial strip, a $\Delta Z$ value equal to zero is assumed.

- The mean velocity of a gravity-driven flow in rough open channels and rivers can be estimated using the Manning's formula (Le Méhauté, 1976), which embodies a large amount of experimental results and can be written as

$v=\frac{1}{m} s^{1 / 3} R^{2 / 3}$

where $v\left(\mathrm{~m} \mathrm{~s}^{-1}\right)$ is the mean velocity of the flow, $s$ $\left(\mathrm{m} \mathrm{m}^{-1}\right)$ is the slope of the channel, $R(\mathrm{~m})$ is the hydraulic radius of the cross section of the channel (defined as the area of the cross section of the channel, $A$, 


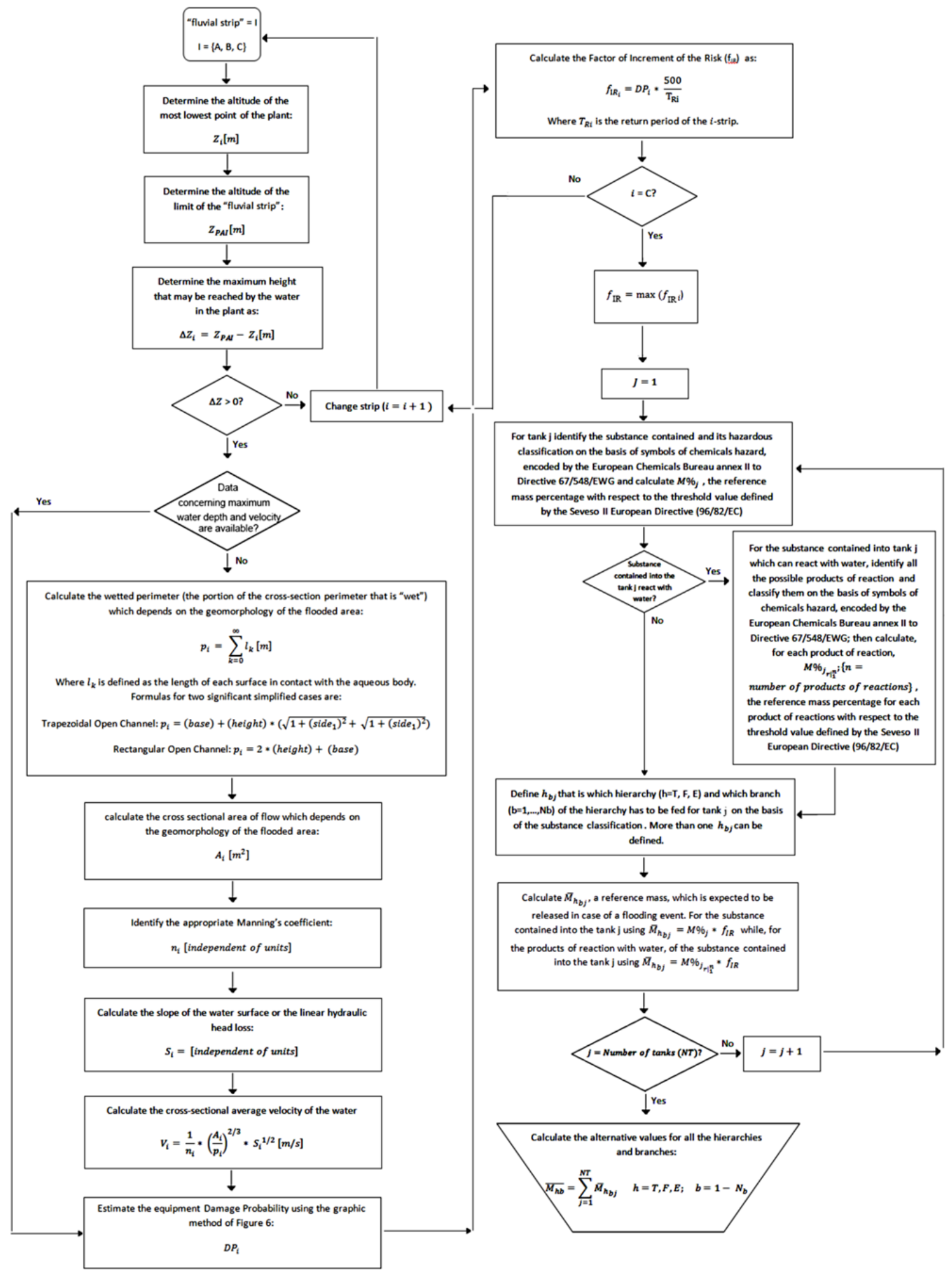

Fig. 4. Procedure for the evaluation of alternatives in the hierarchy. 
a)

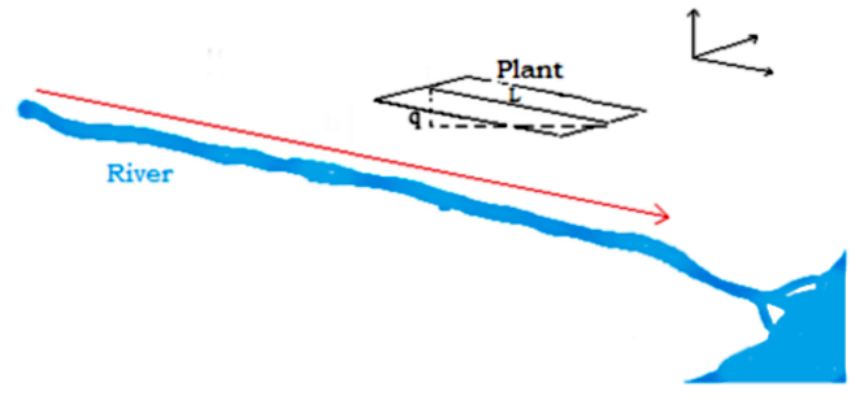

b)

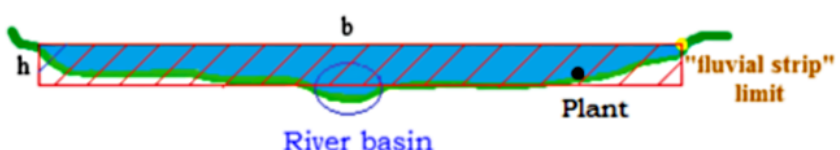

c)

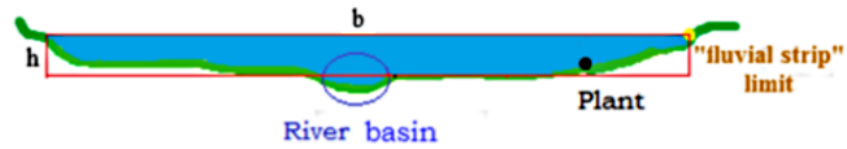

Fig. 5. Graphic representation for the estimation of the (a) slope of the channel $(s),(\mathbf{b})$ cross section of the channel $(A)$ and $(\mathbf{c})$ wetted perimeter $(p)$.

divided by the length of the wetted perimeter, $p$, which can be easily computed assuming a simplified rectangular or trapezoidal shape of the channel), and $n$ is a roughness coefficient related, through standard values, to the river characteristics (Le Méhauté, 1976).

With reference to Fig. 5, the slope of the channel, the cross section of the channel and the wetted perimeter can be defined as follow:

- the slope of the channel can be estimated as the quote difference between the upstream side and downstream side of the plant, $q$, divided by the length of the plant in the direction of the river flow, $L$ :

$s=\frac{q}{L}$

- the cross section of the channel is defined as a rectangular and vertical area transverse to the river passing through the site of interest along the shortest distance between the plant and the river bed. The flow section has as its basis, $b$, the points that limit along that line the fluvial strip to which the plant belongs and as height, $h$, the quote difference between the limits of the fluvial strip and the surface of the river:

$A=b \times h$

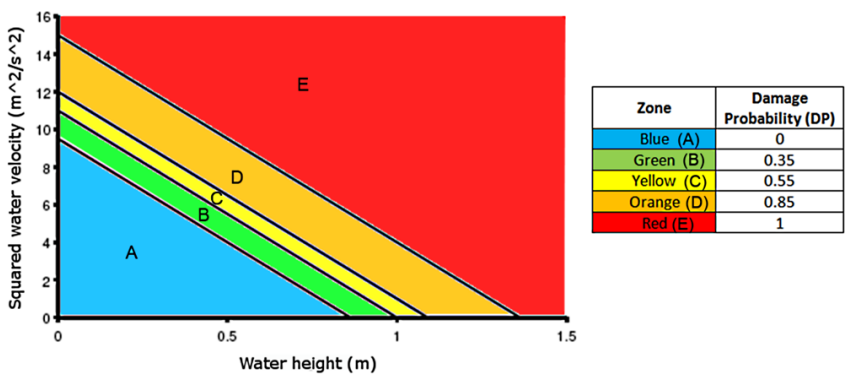

Fig. 6. Estimation of equipment damage probability with respect to maximum water height and velocity.

- The wetted perimeter, $p$, is the perimeter of the flow section and is calculated as

$$
p=2 b+2 h \text {. }
$$

Once the magnitude of the event has been determined in terms of maximum water depth and water velocity, the damage probability of the equipment present in the plant has to be evaluated. The quality of the data reported in industrial accident databases for equipment damage due to floods is usually not homogeneous; in particular, the information about the type of flood and the water impact modes is frequently not available (Cozzani et al., 2010; Fendler, 2008; Krausmann and Mushtaq, 2008). With respect to the structural damage of process equipment caused by the flood, in most cases the reference to equipment damage is only expressed in general terms, without specifying which damage modes are leading to loss of containment (LOC) (Cozzani et al., 2010; Fendler, 2008; Krausmann and Mushtaq, 2008). Consequently, it is difficult to understand the dynamics of the accidents and to assess the extent of the damage suffered by the equipment. As a first approximation, equipment vulnerability may be estimated using the data provided in Fig. 6, which reports in a simple and effective way the equipment damage probability as a function of water height and water velocity (Antonioni et al., 2009). Along the same line discussed elsewhere for earthquake-related Na-Tech events (Busini et al., 2011), since historical analytical methods reveal that storage tanks are the most likely to produce dramatic accidental scenarios in the case of industrial accidents triggered by natural events, reactors, pumps, pipes, and other items are overlooked.

Once the damage probability for the tank $i\left(\mathrm{DP}_{i}\right)$ present in the plant is estimated, the factor of increment of the risk $\left(f_{\mathrm{IR}}\right)$ can be calculated multiplying the damage probability for tank $i$ with the ratio between the $T_{\mathrm{R}}$, for the "fluvial strip" C, e.g. equal to $500 \mathrm{yr}$, and the $T_{\mathrm{R} i}$ of the "fluvial strip" in which the plant is located.

$$
f_{\mathrm{IR} i}=\mathrm{DP}_{i} \times \frac{500}{R_{\mathrm{R} i}}
$$


This accounts for the different probability of floods in different "fluvial strips", leading for "fluvial strip" C to a $f_{\mathrm{IR}}=\mathrm{DP} i$.

Obviously, if a plant is located in "fluvial strip" A (which is characterized by the highest probability), it is subject to all the floods that can take place in that area, from those that involve "fluvial strip" A to those that involve "fluvial strip" C. Consequently, a recursive block is inserted into the flow diagram of Fig. 4 to account for this; however, among the different values of $f_{\mathrm{IR}}$ that can be obtained, the largest one is used for the following calculations, therefore leading to conservative results: $f_{\mathrm{IR}}=\left.\max \left(f_{\mathrm{IR} i}\right)\right|_{i=A, B, C}$.

Only tanks containing liquids with density lower than water are considered since ignition, atmospheric gas dispersion or explosion of subwater liquids is extremely unlikely. Note that, even though in several flood related Na-Tech events, environmental pollution was one of the main consequences; it was not considered in the present paper because of the necessity to validate the method with available QRAs that do not take this issue into account.

The material contained in the various tanks of the plant has to be classified according to its hazardous properties. This can be done according to the European Chemicals Bureau (The Council of the EEC, 1967), which has classified substances in various classes depending on their properties. Toxic substances, classified as $\mathrm{T}+, \mathrm{T}, \mathrm{C}, \mathrm{Xi}, \mathrm{Xn}, \mathrm{N}$, will feed the $\mathrm{KHI}_{\mathrm{T}}$ hierarchy, flammable substances classified as $\mathrm{F}+$ or $\mathrm{F}$ will feed the $\mathrm{KHI}_{\mathrm{F}}$ and $\mathrm{KHI}_{\mathrm{E}}$ hierarchy, while pressurized gaseous substances and explosive substances will fed the $\mathrm{KHI}_{\mathrm{E}}$ hierarchy.

In case of flooding, it is also important to take into account those types of substances that in normal condition do not lead to risk for human safety and health but can become dangerous in contact with water. Thus the block "Substance contained in tank $j$ reacts with water?" is inserted into the logical procedure of Fig. 4. It follows that if the substance reacts with water, the kind and quantities of dangerous byproducts must be estimated assuming that all the chemicals stored in the tank will lead to the stoichiometric amounts of the hazardous byproducts, which will be treated as they were substances contained in the plant.

For each tank $j$ containing a dangerous material or a material which can react dangerously with water, a reference mass expected to be released in case of flood $\left(\overline{M_{h b j}}\right)$ can be computed from its overall factor of increment of the risk $\left(f_{\mathrm{IR}}\right)$ and a relative mass $M_{\% j}$ as

$\overline{M_{h b j}}=M \% j \times f_{\mathrm{IR}}$.

The relative mass percentage contained in tank $j$ is computed as the percentage with respect to the threshold value defined by the Seveso II European Directive (The Council of the EU, 1996). If the directive does not indicate a threshold for the material in the tank, it will not be considered in the analysis.
Table 1. Data used to characterize the magnitude of the natural event and the resulting damage probability (DP) and factor of increment of the risk $\left(f_{\mathrm{IR}}\right)$ for the case study considered.

\begin{tabular}{ll}
\hline "fluvial strip" & $\mathrm{C}$ \\
Return Time & $500($ years $)$ \\
$Z_{\mathrm{PAI}}$ & $10(\mathrm{~m})$ \\
$Z_{\mathrm{i}}$ & $9(\mathrm{~m})$ \\
$\Delta Z$ & $1(\mathrm{~m})$ \\
$p$ & $2504(\mathrm{~m})$ \\
$\mathrm{A}$ & $2500\left(\mathrm{~m}^{2}\right)$ \\
$n$ & 0.035 \\
$s$ & 0.0091 \\
$v$ & $2.721\left(\mathrm{~m} \mathrm{~s}^{-1}\right)$ \\
$\mathrm{DP}$ & $100 \%$ \\
$f_{\mathrm{IR}}$ & 1 \\
\hline
\end{tabular}

The subscript $h b j$ indicates which hierarchy $(h=\mathrm{T}, \mathrm{F}, \mathrm{E})$ and which branch $\left(b=1, \ldots, N_{b h}\right)$ of the hierarchy has to be utilized for tank $j$ on the basis of the substance classification. More than one $h b j$ can be defined for each tank depending on the substances stored.

Once a reference mass is computed for all the tanks present in the plant, the alternative values for all the hierarchies and branches $\left(\overline{M_{h b}}\right)$ can be computed as follows:

$\overline{M_{h b}}=\sum_{j=1}^{\mathrm{NT}} \bar{M}_{h b j} \quad h=\mathrm{T}, \mathrm{F}, \mathrm{E} b=1, \ldots, N_{b h}$

where $N_{b h}$ is the number of branches in the $h$-th hierarchy.

Recalling that the AHP would require to normalize the scores assigned to the alternatives to the maximum value that they can assume in order to obtain a value ranging between $0-1$; it may happen that $\overline{M_{h b}}$ will be higher than 1 ; in this case it will be necessary to limit that value to 1 .

\section{Validation of the methodology}

The study cases used for validating the proposed simplified methodology refers to industrial plants for which results from a detailed QRA considering also Na-Tech events are available in the literature (Campedel et al., 2008a; Antonioni et al., 2009). This allows a comparison between the results of the proposed simplified methodology and the results of a much more detailed QRA.

Finally, a third case study was performed applying the methodology on a well-known real case: flood at Spolana a.s., Neratovice, Czech Republic in August 2002.

\subsection{Case study 1}

The plant layout sketched in Fig. 7 shows the position, number, and catch basins of the units considered in the analysis. According to design standards, each unit is identified by an identification code (e.g. PV_A1, PV_B1, etc.). 


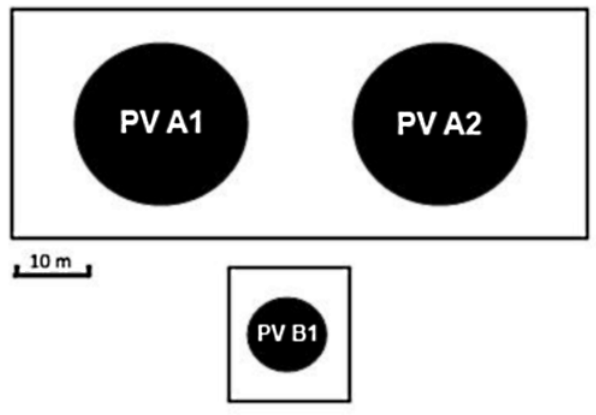

PV C1

Fig. 7. Layout of the plant considered in case study 1.

The data required to characterize the magnitude of the natural event are summarized in Table 1. From these data the damage probability of the equipment and the relative frequency of damage can be easily estimated and are also reported in Table 1.

Considering that in the plant only anhydrous ammonia is present, whose limits are not explicitly defined in the Seveso II Directive, it was considered as a toxic material and Table 2 reports the percentage in respect to the limit of the Directive $(M \%)$ and the index of weighted mass $\left(\overline{M_{h b_{j}}}\right)$ calculated for each tank. The alternative value $\overline{M_{h b}}$ can be easily computed from these data and it is also reported in Table 2.

Although anhydrous ammonia reacts with water, the reaction products have similar chemical properties as ammonia, therefore, leading to similar hazards.

Feeding the various hierarchies with this values, the values of the KHIs can be easily computed through algebraic manipulations (Saaty, 2000) and they are summarized in Table 3 .

The index for fires $\left(\mathrm{KHI}_{\mathrm{F}}\right)$ results from the fact that the substance has low enthalpy of combustion and it is stored as compressed/liquefied gas; the $\mathrm{KHI}_{\mathrm{T}}$ results from the combination of the weight of a substance with low toxicity stored as compressed/liquefied gas; the $\mathrm{KHI}_{\mathrm{E}}$ is determined by the sum of the contribution of a VCE for a substance with low enthalpy of combustion stored as compressed/liquefied gas and a physical expansion in condition of low internal pressure.

To validate the methodology, the overall risk index derived from the qualitative analysis $\left(\mathrm{KHI}_{\mathrm{G}}=6.1 \times 10^{-2}\right.$, meaning "medium" risk) was compared with the numerical values obtained from the QRA. In particular the potential life loss (PLL) Index, which indicates the number of expected deaths in a year in a certain portion of land surrounding the plant, caused by a certain accidental event, it is defined as
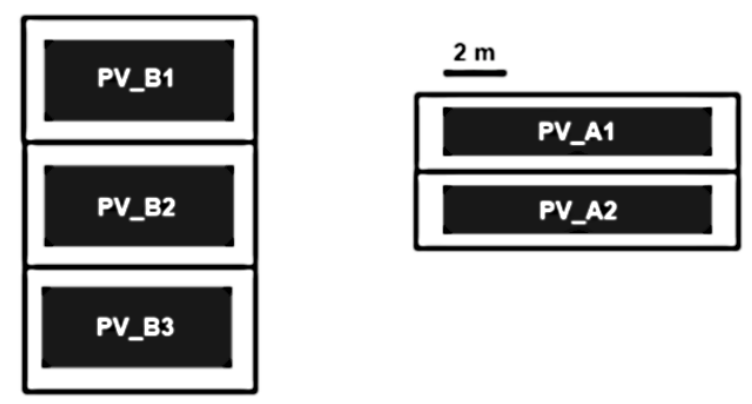

Fig. 8. Layout of the plant considered in case study 2.

$\mathrm{PLL}=\int_{0}^{\infty} F \times d N$

where $F$ is the cumulative frequency of accidents and $N$ is the expected number of fatalities (Antonioni et al., 2009, 2007; Campedel et al., 2008b; Lees, 1996).

The PLL index values obtained from the QRA are summarized in Table 3: the first one does not consider the flood event as initiator event for the industrial accident ( $\mathrm{PLL}_{\text {internal }}$ ) and the second one considers the flood as the initiator event

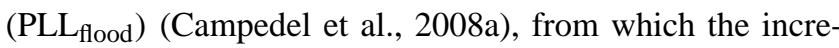
ment of the risk due to the presence of the natural phenomenon can be estimated as

$\mathrm{PLL}_{\text {ratio }}=\frac{\text { PLL flood }_{\text {flol }}}{\text { PLL }}$.

The rank obtained with the proposed shortcut method is in good agreement with the results obtained from the QRA: as discussed elsewhere for earthquake-related $\mathrm{Na}$-Tech events (Busini et al., 2011), the presence of the flood-related NaTech event entails an increment of one order of magnitude of the PLL value, and accordingly the proposed simplified method provides a medium ranking.

\subsection{Case study 2}

The plant layout sketched in Fig. 8 show the position, number, and catch basins of the units considered in the analysis. According to design standards, each unit is identified by an identification code.

On the basis of available data for the site (a flat zone in the north of Italy), the reference flood event was assumed to have a maximum water height of $1 \mathrm{~m}$ and a negligible flow velocity. The expected return time of the flood was considered to be of $1000 \mathrm{yr}$. The data required to characterize the magnitude of the natural event in order to apply the presented shortcut methodology were taken from a more detailed QRA (Campedel et al., 2008a) and are summarized in Table 4. From these data the damage probability of the equipment and the relative frequency of damage can be easily estimated and are also reported in Table 4. 
Table 2. Quantity of substance stored and corresponding index of weighted mass, used in the case-study.

\begin{tabular}{lrrl}
\hline Tank & Quantity stored $\left(10^{3} \mathrm{~kg}\right)$ & $M \%_{j}$ & $\overline{M_{h b_{j}}}$ \\
\hline PV-A1 & 16.8 & 0.084 & $M \%_{j} \times f_{\mathrm{IR}}=0.084$ \\
PV-A2 & 16.8 & 0.084 & $M \%_{j} \times f_{\mathrm{IR}}=0.084$ \\
PV-B & 2.8 & 0.014 & $M \%_{j} \times f_{\mathrm{IR}}=0.014$ \\
PV-C & 5.6 & 0.028 & $M \%_{j} \times f_{\mathrm{IR}}=0.028$ \\
\hline \multicolumn{2}{l}{ Total index of weighted mass $\overline{M_{h b}}=\sum_{j=1}^{4} \overline{M_{h b_{j}}}$} & 0.21 \\
\hline
\end{tabular}

Table 3. Risk indexes obtained from QRA (PLLs [28]) and from the presented methodology (KHIs) for case study 1.

\begin{tabular}{llllllll}
\hline $\begin{array}{l}\mathrm{PLL}_{\text {internal }} \\
\text { (fatalities/year) }\end{array}$ & $\begin{array}{l}\mathrm{PLL}_{\text {flood }} \\
\text { (fatalities/year) }\end{array}$ & $\mathrm{PLL}_{\text {ratio }}$ & $\mathrm{KHI}_{\mathrm{F}}$ & $\mathrm{KHI}_{\mathrm{T}}$ & $\mathrm{KHI}_{\mathrm{E}}$ & $\mathrm{KHI}_{\mathrm{G}}$ & Risk level \\
\hline $7.7 \times 10^{-5}$ & $3.5 \times 10^{-3}$ & $4.7 \times 10^{1}$ & $2.6 \times 10^{-2}$ & $2.3 \times 10^{-2}$ & $5.0 \times 10^{-2}$ & $6.1 \times 10^{-2}$ & Medium \\
\hline
\end{tabular}

Table 4. Data used to characterize the magnitude of the natural event and the resulting damage probability (DP) and factor of increment of the risk $\left(f_{\mathrm{IR}}\right)$ for the case study considered.

\begin{tabular}{ll}
\hline "fluvial strip" & $\mathrm{C}$ \\
Return Time & 1000 (years) \\
$\Delta Z$ & $1(\mathrm{~m})$ \\
$v$ & $0\left(\mathrm{~m} \mathrm{~s}^{-1}\right)$ \\
$\mathrm{DP}$ & $55 \%$ \\
$f_{\mathrm{IR}}$ & 0.275 \\
\hline
\end{tabular}

In the plant only anhydrous ammonia is present: Table 5 reports the percentage respect to the limit of the Seveso II Directive $(M \%)$ and the index of weighted mass $\left(\overline{M_{h b_{j}}}\right)$ calculated for each tank. The alternative value $\overline{M_{h b}}$ can be easily computed from these data and it is also reported in Table 5. As in the case study 1 , for the ammonia the limits of the toxics were considered and the reaction products were neglected having chemical properties similar to the ammonia.

Feeding the various hierarchies with these values, the values of the KHIs can be easily computed through algebraic manipulations (Saaty, 2000); their values are summarized in Table 6.

The parameters that contribute to define the indexes results from the fact that the substance has low enthalpy of combustion and it is stored as compressed/liquefied gas $\left(\mathrm{KHI}_{\mathrm{F}}\right)$, is considered to have a low level of toxicity $\left(\mathrm{KHI}_{\mathrm{T}}\right)$, and it may give rise to a VCE and to a physical expansion in condition of low internal pressure $\left(\mathrm{KHI}_{\mathrm{E}}\right)$.

To validate the methodology, the overall risk index derived from the qualitative analysis $\left(\mathrm{KHI}_{\mathrm{G}}=2.5 \times 10^{-2}\right.$, meaning "medium" risk) is compared with the numerical values obtained from the QRA (Campedel et al., 2008a).

The PLL index values obtained from the QRA are summarized in Table 6: the first one does not considers the flood event as initiator event for the industrial accident ( $\left.P L L_{\text {internal }}\right)$
Table 5. Quantity of substance stored and corresponding index of weighted mass used in the case-study.

\begin{tabular}{lrrr}
\hline Tank & $\begin{array}{r}\text { Quantity stored } \\
\left(10^{3} \mathrm{~kg}\right)\end{array}$ & $M \%_{j}$ & $\overline{M_{h b_{j}}}$ \\
\hline PV-A1 & 15.14 & 0.076 & 0.021 \\
PV-A2 & 15.14 & 0.076 & 0.021 \\
PV-B1 & 11 & 0.055 & 0.015 \\
PV-B2 & 11 & 0.055 & 0.015 \\
PV-B3 & 11 & 0.055 & 0.015 \\
\hline \multicolumn{2}{l}{ Total index of weighted mass $\overline{M_{h b}}$} & 0.087 \\
\hline
\end{tabular}

and the second one considers the flood as the initiator event

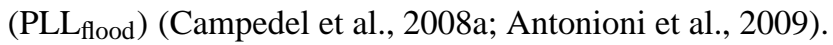

The rank obtained with the proposed shortcut method is in good agreement with the results obtained from the QRA: as discussed elsewhere for earthquake-related Na-Tech events (Busini et al., 2011), the presence of the flood-related NaTech event entails an increment of one order of magnitude of the PLL value, and accordingly the proposed simplified method provides a medium ranking.

\subsection{Case study 3: flood at Spolana a.s., Neratovice, Czech Republic in August 2002}

Spolana is a Czech chemical plant in Neratovice established in 1898. The chemical plant is situated approximately $25 \mathrm{~km}$ north of Prague at the confluence of the Košátecký brook and the river Elbe. In 2002 the river Elbe overflowed and flooded the Spolana chemical plant, causing a release of chlorine of about $400 \mathrm{~kg}$. It was a catastrophic flood with water exceeding the "hundred year water level" by $1.3 \mathrm{~m}$ (Hudec and Luks, 2004).

The Spolana site consists of more than 700 separate premises (350 of which are buildings); at the time of the flood 
Table 6. Risk indexes obtained from QRA (PLLs [28]) and from the presented methodology (KHIs) for case study 1.

\begin{tabular}{llllllll}
\hline $\begin{array}{l}\mathrm{PLL}_{\text {internal }} \\
\text { (fatalities/year) }\end{array}$ & $\begin{array}{l}\mathrm{PLL}_{\text {flood }} \\
\text { (fatalities/year) }\end{array}$ & $\mathrm{PLL}_{\text {ratio }}$ & $\mathrm{KHI}_{\mathrm{F}}$ & $\mathrm{KHI}_{\mathrm{T}}$ & $\mathrm{KHI}_{\mathrm{E}}$ & $\mathrm{KHI}_{\mathrm{G}}$ & Risk level \\
\hline $8.0 \times 10^{-4}$ & $1.9 \times 10^{-2}$ & $2.5 \times 10$ & $1.1 \times 10^{-2}$ & $1.0 \times 10^{-2}$ & $2.1 \times 10^{-2}$ & $2.5 \times 10^{-2}$ & Medium \\
\hline
\end{tabular}

Table 7. List of tanks divided by storehouse (a, b, c), quantity of substance stored for each tank, and corresponding index of weighted mass used in the case-study 3 .

\begin{tabular}{lrrr}
\hline & $\begin{array}{r}\text { Quanity stored } \\
\left(10^{3} \mathrm{~kg}\right)\end{array}$ & $M \%$ & $\overline{M_{h b_{j}}}$ \\
& 102.4 & 409.6 & 409.6 \\
\hline PV_A1 (full 80\%) & 3.84 & 15.36 & 15.36 \\
PV_A2 (3\%) & 3.84 & 15.36 & 15.36 \\
PV_A3 (3\%) & 3.84 & 15.36 & 15.36 \\
PV_A4 (3\%) & 3.84 & 15.36 & 15.36 \\
PV_A5 (3\%) & 25.6 & 102.4 & 102.4 \\
PV_A6 (20\%) & 25.6 & 102.4 & 102.4 \\
PV_A7 (20\%) & 25.6 & 102.4 & 102.4 \\
PV_A8 (20\%) & 25.6 & 102.4 & 102.4 \\
PV_A9 (20\%) & 25.6 & 102.4 & 102.4 \\
PV_A10 (20\%) & 20.48 & 81.92 & 81.92 \\
PV_B1 (full 80 \%) & \multicolumn{3}{c}{1} \\
\hline Total index of weighted mass (limited to 1) $\overline{M_{h b}}$ & 1 \\
\hline
\end{tabular}

Spolana had approximately 2500 employees including shiftworkers.

Liquefied chlorine was stored in chlorine tanks located in two storehouses, whose internal arrangement was practically identical. There were five tanks of $80 \mathrm{~m}^{3}$ located in each building plus another smaller tank (blow-case) of $16 \mathrm{~m}^{3}$ situated in the old storehouse (Hudec and Luks, 2004). The tanks in the chlorine storehouses contained different amounts of chlorine: some contained only gaseous chlorine as a residue after discharging, some were filled up to $20 \%$, and one tank was practically full (Hudec and Luks, 2004). Since precise information about how many tanks were filled and in which way are unknown, in our calculation we will assume, for conservative reasons, that only 4 tanks were filled at $3 \%$ (those that contained only gaseous chlorine as a residue after discharging); 5 tanks were filled at $20 \%$ and the one full was filled at $80 \%$ (full). Moreover, the $16 \mathrm{~m}^{3}$ blow-case tank was assumed to be full for conservative reasons $(80 \%)$ leading to chlorine quantities much larger than those defined in Article 8 of Seveso II Directive.

From the flood record it can be deduced that the flood in its critical stage (at 15:00 on the day 15 August 2002) reached $164.5 \mathrm{~m}$ a.s.l. (Hudec and Luks, 2004). Considering that the lowest point of the plant is sited about $162 \mathrm{~m}$ a.s.l. we can consider a maximum water level of about $2.5 \mathrm{~m}$. Moreover, on the basis of available data for the site (Hudec and Luks, 2004), the reference flood event was assumed to have a negligible flow velocity and the expected return time of the flood
Table 8. Risk indexes obtained from the presented methodology (KHIs) for case study 3.

\begin{tabular}{lrrrr}
\hline $\mathrm{KHI}_{\mathrm{F}}$ & $\mathrm{KHI}_{\mathrm{T}}$ & $\mathrm{KHI}_{\mathrm{E}}$ & $\mathrm{KHI}_{\mathrm{G}}$ & Risk level \\
\hline 0.00 & $4.44 \times 10^{-1}$ & $2.18 \times 10^{-1}$ & $4.95 \times 10^{-1}$ & $\mathrm{HIGH}$ \\
\hline
\end{tabular}

was assumed equal to $500 \mathrm{yr}$, since the expected one-hundred years flood was broadly exceeded $(1.3 \mathrm{~m})$ (Hudec and Luks, 2004); this choice is not determinant because the graph in Fig. 6 shows that for water levels higher than $1.4 \mathrm{~m}$ the damage probability is $100 \%$, regardless water flow velocity.

Considering that in the plant only liquefied chlorine is present, Table 7 reports the percentage respect to the limit of the Seveso II Directive $(M \%)$ and the index of weighted mass $\left(\overline{M_{h b_{j}}}\right)$ calculated for each tank. The alternative value $\overline{M_{h b}}$ can be easily computed from these data and it is also reported in Table 7.

Feeding the various hierarchies with $\overline{M_{h b}}$ value, the values of the KHIs can be easily computed through algebraic manipulations (Saaty, 2000); their values are summarized in Table 8 . The parameters that contribute to define the indexes arise from the liquefied chlorine properties: not flammable $\left(\mathrm{KHI}_{\mathrm{F}}=0\right)$, stored at low pressure $\left(\mathrm{KHI}_{\mathrm{E}}>0\right)$ and highly toxic $\left(\mathrm{KHI}_{\mathrm{T}}>0\right)$.

The high risk to flood-related Na-tech events predicted by the methodology is in good agreement whit the large consequences observed. In fact, after being flooded, the empty and less loaded tanks were lifted by buoyancy forces according to Archimedes' law and displaced from their normal positions. The buoyancy forces were so strong that their action deformed and lifted the walkways situated above the tanks. The closing valves in the full tank got caught by the walkway and were completely torn off as the walkway kept moving upwards. As a consequence of the valves being torn off from the full tank, a massive leakage of chlorine occurred (Hudec and Luks, 2004). The impact of the flood at the chemical factory resulted not only in a contamination of the River Elbe but also in a chlorine cloud which forced residents to seek safety indoors.

\section{Conclusions}

Since floods may be powerful and prominent mechanisms of direct and indirect hazmat releases, flood related $\mathrm{Na}$-Tech events might increase the impact and the overall damage in surrounding areas of industrial sites. 
The aim of this study was to provide a referential shortcut methodology for the assessment of industrial risks induced by floods through suitable qualitative KHIs computed using the analytical hierarchy process.

The methodology developed requires few resources and little information on both the plant and the expected flood. Therefore, it would be suitable at any stage of a plant's life (i.e. from the early design stage to an already existing plant).

The developed methodology was validated by a comparison with independent results obtained by QRA reports (Campedel et al., 2008a; Antonioni et al., 2009) in terms of the ratio between PLL values in presence and absence of the flood event, showing a good agreement. Moreover, it was also applied on a well-known real case: flood at Spolana a.s., Neratovice, Czech Republic in August 2002 leading to results in good agreement with the recorded damages.

Acknowledgements. The research leading to these results has received funding from the FP7, the European Union's 7th Framework Programme for Research and Technological Development (FP7/2007-2013) under grant agreement no. 213345. Moreover, the financial support of the Italian MIUR - PRIN2007 is gratefully acknowledged.

Edited by: F. Castelli

Reviewed by: two anonymous referees

\section{References}

Antonioni, G., Spadoni, G., and Cozzani, V.: A methodology for the quantitative risk assessment of major accidents triggered by seismic events, J. Hazard. Material., 147, 48-59, doi:10.1016/j.jhazmat.2006.12.043, 2007.

Antonioni, G., Bonvicini, S., Spadoni, G., and Cozzani, V.: Development of a framework for the risk assessment of Na-Tech accidental events, Reliab. Eng. Syst. Safe., 94, 1442-1450, doi:10.1016/j.ress.2009.02.026, 2009.

Autorità di bacino del fiume Po: Progetto di Piano stralcio per l'Assetto Idrogeologico (PAI), 7. Norme di attuazione, Titolo II Norme per le fasce fluviali, Allegato 3 - Metodo di delimitazione delle fasce fluviali, 1999.

Bell, R. and Glade, T.: Multi-hazard analysis in natural risk assessments, in: Risk Analysis Iv, edited by: Brebbia, C. A., Management Information Systems, Wit Press, Southampton, 197-206, 2004.

Busini, V., Marzo, E., Callioni, A., and Rota, R.: Definition of a short-cut methodology for assessing earthquakerelated Na-Tech risk, J. Hazard. Material., 192, 329-339, doi:10.1016/j.jhazmat.2011.05.022, 2011.

Campedel, M., Antonioni, G., Cozzani, V., and Di Baldassarre, G.: A framework for the assessment of the industrial risk caused by floods, ESREL Conference 2008, 2008a.

Campedel, M., Cozzani, V., Garcia-Agreda, A., and Salzano, E.: Extending the quantitative assessment of industrial risks to earthquake effects, Risk Anal., 28, 1231-1246, doi:10.1111/j.15396924.2008.01092.x, 2008b.

Chang, J. I. and Lin, C.-C.: A study of storage tank accidents, J. Loss Prevent. Process Ind., 19, 51-59, 2006.
Council Directive 67/548/EEC: available at: http://eur-lex.europa. eu/LexUriServ/LexUriServ.do?uri=_CELEX:31967L0548:en: NOT (last access: 17 October 2011), 1967.

COUNCIL DIRECTIVE 96/82/EC: available at: http: //eur-lex.europa.eu/LexUriServ/LexUriServ.do?uri=_CELEX: 31996L0082:EN:HTML (last access: 20 October 2011), 1996.

Cozzani, V., Bandini, R., Basta, C., and Christou, M. D.: Application of land-use planning criteria for the control of major accident hazards: A case-study, J. Hazard. Material., 136, 170-180, doi:10.1016/j.jhazmat.2005.12.031, 2006.

Cozzani, V., Campedel, M., Renni, E., and Krausmann, E.: Industrial accidents triggered by flood events: Analysis of past accidents, J. Hazard. Material., 175, 501-509, doi:10.1016/j.jhazmat.2009.10.033, 2010.

Cruz, A. M. and Krausmann, E.: Hazardous-materials releases from offshore oil and gas facilities and emergency response following Hurricanes Katrina and Rita, J. Loss Prevent. Proc. Ind., 22, 5965, doi:10.1016/j.jlp.2008.08.007, 2009.

Cruz, A. M. and Okada, N.: Methodology for preliminary assessment of Natech risk in urban areas, Nat. Hazards, 46, 199-220, doi:10.1007/s11069-007-9207-1, 2008.

Cruz, A. M., Steinberg, L. J., and Vetere-Arellano, L.: Emerging issues for natech disaster risk management in Europe, J. Risk Res., 9, 483-501, doi:10.1080/13669870600717657, 2006.

Fabbrocino, G., Iervolino, I., Orlando, F., and Salzano, E.: Quantitative risk analysis of oil storage facilities in seismic areas, J. Hazard. Material., 123, 61-69, doi:10.1016/j.jhazmat.2005.04.015, 2005.

Fendler, R.: Floods and safety of establishments and installations containing hazardous substances, Nat. Hazards, 46, 257-263, doi:10.1007/s11069-007-9209-z, 2008.

Galderisi, A., Ceudech, A., and Pistucci, M.: A method for na-tech risk assessment as supporting tool for land use planning mitigation strategies, Nat. Hazards, 46, 221-241, doi:10.1007/s11069008-9224-8, 2008.

Hudec, P. and Luks, O.: Flood at Spolana a.s. in August 2002, Loss Prevention Bulletin (LPB), Sample papers, LPB180, 2004, 3639, 2004.

Krausmann, E. and Mushtaq, F.: A qualitative Natech damage scale for the impact of floods on selected industrial facilities, Nat. Hazards, 46, 179-197, doi:10.1007/s11069-007-9203-5, 2008.

Krausmann, E., Cozzani, V., Salzano, E., and Renni, E.: Industrial accidents triggered by natural hazards: an emerging risk issue, Nat. Hazards Earth Syst. Sci., 11, 921-929, doi:10.5194/nhess11-921-2011, 2011.

Le Méhauté, B.: An Introduction to Hydrodynamics \& Water Waves, Springer-Verlag, New York, 1976.

Lees, F. P.: Lees' Loss Prevention in the Process Industries, edited by: Mannan, S., 3rd Edn., Elsevier, 1996.

Picou, J. S.: Katrina as a Natech Disaster, University of South Alabama, 2009.

Presidenza del Consiglio dei Ministri: Atto di indirizzo e coordinamento per l'individuazione dei criteri relativi agli adempimenti di cui all' art. 1, commi 1 e 2, del decreto-legge 11 giugno 1998, n. 180, Consiglio Nazionale dei Geologi, 29 settembre 1998 (G.U. 5 gennaio 1999 n.3), 1998.

Rasmussen, K.: Natural events and accidentswith hazardous materials, J. Hazard. Materials, 40, 43-54, 1995. 
Renni, E., Basco, A., Busini, V., Cozzani, V., Krausmann, E., Rota, R., and Salzano, E.: Awareness and mitigation of NaTech accidents: Toward a methodology for risk assessment, in: Cisap4: 4th International Conference on Safety \& Environment in Process Industry, edited by: Buratti, S. S., Chemical Engineering Transactions, Aidic Servizi Srl, Milano, 383-389, 2010.

Rota, R., Caragliano, S., Manca, D., and Brambilla, S.: A Short cut methodology for flood-technological risk assessment, in: Chemical Engineering Transactions, CISAP3 - 3rd International Conference on Safety \& Environment in Process Industry Rome, 5360, 2008.
Saaty, T.: Fundamentals of Decision Making and Priority Theory With the Analytic Hierarchy Process, Analytic Hierarchy Process Series, 2000

Showalter, P. S. and Myers, M. F.: Natural disasters in the UnitedStates as release agents of oil, chemicals, or radiological materials between 1980-1989 - Analysis and recommendations, Risk Anal., 14, 169-182, 1994.

Young, S., Balluz, L., and Malilay, J.: Natural and technologic hazardous material releases during and after natural disasters: a review, Sci. Total Environ., 322, 3-20, doi:10.1016/s00489697(03)00446-7, 2004. 